\title{
Spectral and correlation properties of rings of delay-coupled elements: Comparing linear and nonlinear systems
}

\author{
O. D'Huys, ${ }^{1,2}$ I. Fischer, ${ }^{3}$ J. Danckaert, ${ }^{1}$ and R. Vicente ${ }^{4,5}$ \\ ${ }^{1}$ Applied Physics Research Group, Vrije Universiteit Brussel, Pleinlaan 2, 1050 Brussel, Belgium \\ ${ }^{2}$ Institut für Theoretische Physik, Universität Würzburg, Am Hubland, 97074 Würzburg, Germany \\ ${ }^{3}$ Instituto de Fisica Interdisciplinar y Sistemas Complejos (UIB-CSIC), Campus Universitat de les Illes Balears, \\ E-07122 Palma de Mallorca, Spain \\ ${ }^{4}$ Max-Planck-Institute for Brain Research, Deutschordenstraße 46, 60528 Frankfurt am Main, Germany \\ ${ }^{5}$ Frankfurt Institute for Advanced Studies, Ruth-Moufang-Straße 1, 60438 Frankfurt am Main, Germany
}

(Received 10 January 2012; published 15 May 2012)

\begin{abstract}
The dynamical properties of delay-coupled systems are currently of great interest. So far the analysis has concentrated primarily on identical synchronization properties. Here we study the dynamics of rings of delaycoupled nodes, a topology that cannot show identical synchronization, and compare its properties to those of linear stochastic maps. We find that, in the long delay limit, the correlation functions and spectra of delay-coupled rings of nonlinear systems obey the same scaling laws as linear systems, indicating that important properties of the emerging solution result from network topology.
\end{abstract}

DOI: 10.1103/PhysRevE.85.056209 PACS number(s): 05.45.Xt, 02.50.Fz, 89.75.Hc, 02.30.Ks

\section{INTRODUCTION}

Recently, the dynamical properties of networks with delayed interactions have received much interest $[1,2]$. Delayed interactions play an important role in systems as diverse as coupled semiconductor lasers [3,4], population dynamics [5], traffic [6], communication networks [7], genetic circuits [8,9], and the brain [10]. In addition, delayed complex systems have proven to be useful for applications such as encrypted communication [11] or information processing [12].

Considerable research has been devoted to the study of zero-lag synchronization of delay-coupled chaotic networks, i.e., whether, and under which conditions, the nodes show phase or identical synchronization [13-19]. Little has been done analytically to characterize the network dynamics if identical synchronization is unstable. A typical example in this context are two mutually coupled units with delay, which do not show significant correlation at zero lag. Nevertheless, some dynamical properties of nodes in a delay-coupled system can be deduced from those of a single element with delayed feedback. Van der Sande et al. [20] compared numerically the autocorrelation functions and spectral densities of two mutually coupled elements with those of a single element with feedback in the limit of long delays for various chaotic systems including semiconductor lasers and Ikeda and Mackey-Glass oscillators. They found several interesting features: Delayed feedback and delay-coupled systems share the same broadband chaotic envelope in the spectral density, with the discrete delay peaks being smaller in the coupled system. The correspondence of autocorrelation functions was even more striking: The peaks at even multiples of the delay time are reproduced in the autocorrelation function of the coupled system. These results were extended to a unidirectional ring of $N$ elements with constant round trip delay: The autocorrelation function at $\tau$ (round-trip delay) for a ring element corresponds to the autocorrelation function of the single element evaluated at $\tau N$.

However, while these findings were only phenomenological, based on numerical simulations, we demonstrate that such correlation patterns can be derived from linear effects solely.
We show analytically how the dynamical properties of delaycoupled rings of linear stochastic maps are determined from the properties of simple delayed feedback systems. As a main result we find a remarkable correspondence between linear stochastic and nonlinear deterministic networks: Coupled in a unidirectional ring, chaotic systems show, in the limit of long delays, the same correlation pattern as stochastic systems, although the former can show generalized synchronization. Our results therefore imply that important properties of the dynamics of coupled systems are predominantly shaped by the coupling topology.

\section{RINGS OF LINEAR STOCHASTIC MAPS}

We consider a network of $N$ coupled linear stochastic maps

$$
x_{n}(t)=\alpha x_{n}(t-1)+\xi_{n}(t)+\beta \sum_{m}^{N} A_{n m} x_{m}(t-D) .
$$

The parameter $\alpha$ accounts for the system's own dynamics, $\beta$ represents a coupling strength, and the interaction delay is given by $D$. The coupling topology is described by the connection matrix $A$. We consider here ring topologies, i.e., the network is invariant under cyclic permutations of the nodes and the connection matrix is circulant. Important cases include unidirectional rings, bidirectional rings, and all-to-all or globally coupled networks. Each node $x_{n}(t)$ is driven by an independent white noise term $\xi_{n}(t)$. We choose $\alpha$ and $\beta$ such that the network output is wide-sense stationary and thus remains finite. For equal noise strength over the network $\left\langle\xi_{n}(t)^{2}\right\rangle=\sigma^{2}$ the nodes share the same statistical properties such as spectral density, variance, and autocorrelation function.

We are interested in computing the covariance functions between any pair of nodes $r_{l}(k)$,

$$
r_{l}(k)=\left\langle x_{n+l}(t) x_{n}(t-k)\right\rangle .
$$

Therefore, we multiply Eq. (1) by $x_{n-l}(t-k)$ (with $k \geqslant 0$ ) and average over time. We obtain a set of 
equations

$$
\begin{aligned}
r_{l}(k) & =\alpha r_{l}(k-1)+\beta \sum_{m}^{N} A_{n m} r_{m-n+l}(k-D)+\sigma^{2} \delta_{l 0} \delta_{k 0} \\
& =\alpha r_{l}(k-1)+\beta \sum_{m}^{N} A_{l m} r_{m}(k-D)+\sigma^{2} \delta_{l 0} \delta_{k 0},
\end{aligned}
$$

where we used the properties of the circulant matrix $A$. The set of coupled equations (2) provides a unique solution for the covariance functions within the network and the variance of the nodes. Note that the covariance functions scale with the noise strength $\sigma^{2}$; their shape, however, is independent of the noise strength.

Evaluating Eqs. (2) along the eigenvectors of the connection matrix $A\left[v_{n}(k)=\sum_{m} \exp (2 \pi i n m / N) r_{m}(k)\right]$ allows us to uncouple the system and we find

$$
v_{n}(k)=\alpha v_{n}(k-1)+\beta \lambda_{n} v_{n}(k-D)+\sigma^{2} \delta_{k 0},
$$

where $\lambda_{n}$ is the eigenvalue corresponding to the eigenvector $v_{n}$. These equations describe the covariance function of a single node with a complex feedback strength $\lambda_{n} \beta$. Consequently, one can calculate the covariance functions within a ring of $N$ elements from the covariance functions $v_{n}(k)=r\left(k, \lambda_{n} \beta\right)$ of $N$ different single nodes with delayed feedback:

$$
\begin{aligned}
v_{n}(k) & =\sum_{m}^{N} e^{2 \pi i m n / N} r_{m}(k)=r\left(k, \lambda_{n} \beta\right) \\
\Leftrightarrow r_{m}(k) & =\frac{1}{N} \sum_{n}^{N} e^{-2 \pi i m n / N} r\left(k, \lambda_{n} \beta\right) .
\end{aligned}
$$

The autocorrelation function $r\left(k, \lambda_{n} \beta\right)$ of such a single node with feedback strength $\lambda_{n} \beta$ shows extrema at multiples of the delay $m D$. For a wide range of values of $\alpha$ and $\beta$, the height of these peaks is approximated by an exponential decay $\lambda_{n} \beta$, i.e., $r\left(m D, \lambda_{n} \beta\right) \approx\left(\lambda_{n} \beta\right)^{m} r\left(0, \lambda_{n} \beta\right)$. Examples of the autocorrelation functions of single maps with delayed feedback and opposite coupling phases are shown in Figs. 1(a) and 1(c). The variance of the map increases with $\alpha$ and $\left|\lambda_{n}\right| \beta$, but it does not depend on the coupling phase.

On the basis of Eq. (4) and the dynamics of a single element subject to feedback that we just reviewed, it is already possible to predict several features of the coupled dynamics. In particular, for just two coupled elements we have $\lambda_{0}=1$ in the parallel direction and $\lambda_{1}=-1$ in the transverse direction. Thus Eq. (4) translates as

$$
\begin{aligned}
& r_{0}(k)=\frac{1}{2}[r(k, \beta)+r(k,-\beta)], \\
& r_{1}(k)=\frac{1}{2}[r(k, \beta)-r(k,-\beta)] .
\end{aligned}
$$

The autocorrelation function $r_{0}(k)$ is the average of the autocorrelation functions of a map with positive feedback and a map with negative feedback or the average of an in-phase state $x_{1}(t)=x_{2}(t)$ and an antiphase state $x_{1}(t)=-x_{2}(t)$. The peaks located at $k=2 n D$ are positive for both in-phase and antiphase states and they add up to each other. However, the peaks at $k=$ $(2 n+1) D$ cancel each other. For the cross correlation we find the opposite result: The peaks at $k=2 n D$ cancel, while those at $(2 n+1) D$ add up. Thus the cross correlation is maximal for $k= \pm D$ and vanishes at zero lag. These features are illustrated

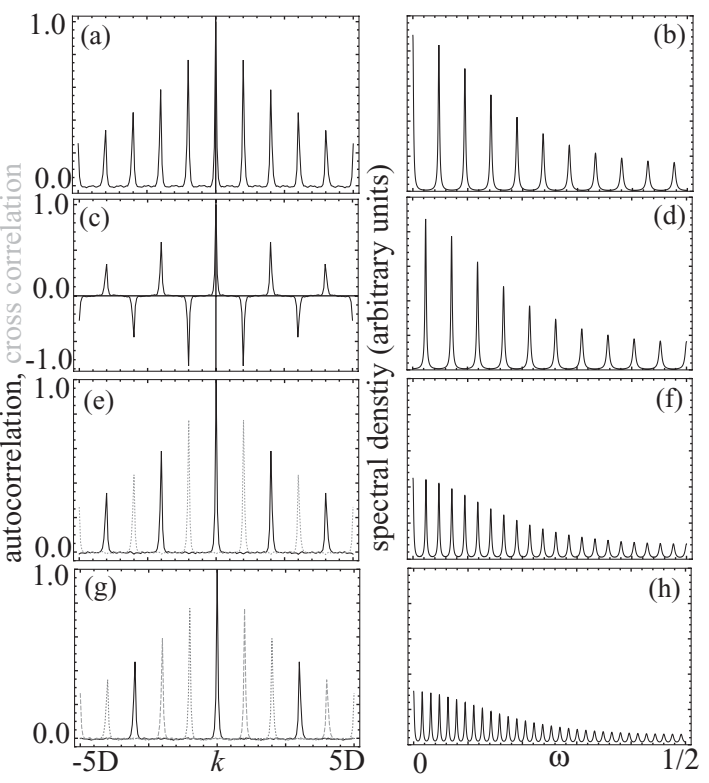

FIG. 1. Autocorrelation (black solid line), cross correlations (gray dashed lines), and the power spectrum $\left|X\left(e^{2 \pi i \omega}\right)\right|^{2}$ of coupled stochastic maps with different numbers of units [modeled by Eq. (1)]. Panels (a) and (b) and panels (c) and (d) illustrate a single map with positive and negative delayed feedback, respectively, and panels (e) and (f) and panels (g) and (h) show the cases of two and three stochastic maps coupled in a unidirectional ring, respectively. The parameters are $\alpha=0.1, \beta= \pm 0.75, D=20$, and $\sigma^{2}=1$. Note that the spectral densities are plotted on a linear scale.

in Fig. 1, which shows the autocorrelations of single maps with positive and negative feedback and the autocorrelation and cross correlation of two coupled stochastic maps.

In the case of a unidirectional ring of $N$ elements we find

$$
\begin{aligned}
& r_{0}(k)=\frac{1}{N}\left[r(k, \beta)+\cdots+r\left(k, e^{-2 \pi i / N} \beta\right)\right], \\
& r_{n}(k)=\frac{1}{N}\left[r(k, \beta)+\cdots+e^{2 \pi i n / N} r\left(k, e^{-2 \pi i / N} \beta\right)\right] .
\end{aligned}
$$

The autocorrelation function of a network element $r_{0}(k)$ has a contribution from an in-phase direction $x_{1}(t)=x_{2}(t)=\cdots=$ $x_{N}(t)$ and contributions from different out-of-phase directions $x_{n}=e^{2 \pi i m / N} x_{n+1}$. The extrema in the autocorrelation at $k=$ $m N D$ add up to each other, the other extrema cancel, and we recover a scaling relation similar to that found numerically by Van der Sande et al. [20]. In the cross correlations $r_{n}(k)$ the extrema located at $k=(m N+n) D$ add up to each other while the other extrema vanish. Moreover, we find that the variance of the nodes does not depend on the number of units since all different contributions have the same variance. The different correlation functions and the spectral densities of rings with one, two, and three elements are shown in Fig. 1. In fact, the more general relation (4) implies that the variance of the networks nodes decreases with the different $\left|\lambda_{n}\right|$ and thus in general with the degree of the nodes, similar to the case of undelayed networks [21].

The spectral density and covariance functions are related to each other by the Wiener-Khinchin theorem; therefore, we expect a scaling relation similar to Eq. (4) for the spectral density of network elements. The calculation is straightforward 
by applying a Z-transform $X_{n}(z)=\sum_{t} x_{n}(t) z^{-t}$ to Eq. (1),

$$
X_{n}(z)=\alpha z^{-1} X_{n}(z)+\beta \sum_{m}^{N} A_{n m} z^{-D} X_{m}(z)+\Xi_{n}(z) .
$$

Here the variable $z$ represents the different frequency components $z=e^{2 \pi i \omega}$ and $\Xi_{n}(z)$ is the frequency spectrum of the noise sources $\xi_{n}(z)$. Along the eigenvectors $V_{m}=$ $\frac{1}{\sqrt{N}} \sum_{n} e^{2 \pi i n m / N} X_{n}$, we find

$$
\begin{aligned}
V_{m}(z) & =\alpha z^{-1} V_{m}(z)+\beta \lambda_{m} z^{-D} V_{n}(z)+\Xi_{m}(z) \\
& \Rightarrow V_{m}(z)=\frac{\Xi_{m}(z)}{1-\alpha z^{-1}-\lambda_{n} \beta z^{-D}},
\end{aligned}
$$

with $\Xi_{m}=\frac{1}{\sqrt{N}} \sum_{n} e^{2 \pi i n m / N} \Xi_{n}$. This leads to a spectral density for the individual nodes

$$
\begin{aligned}
\left|X_{n}(z)\right|^{2} & =\frac{1}{N} \sum_{m}^{N}\left|V_{m}(z)\right|^{2} \\
& =\frac{1}{N} \sum_{m}^{N} \frac{1}{\left|1-\alpha z^{-1}-\lambda_{n} \beta z^{-D}\right|^{2}} .
\end{aligned}
$$

Just like for the autocorrelation function, we find that the spectral density of a network element is the average over its in-phase and out-of-phase states, or an average over different single nodes with a delayed feedback with strength $\lambda_{n} \beta$. Such a single-node spectrum is maximal at frequencies for which $2 \pi \omega D=\arg \left(\lambda_{n}\right)$ holds. The height of the peaks increases with the coupling strength $\left|\lambda_{n}\right| \beta$, while the width of the peaks and the spacing between the peaks depend on the feedback delay $D$.

Consequently, for a unidirectional ring, each mode introduces specific peaks in the spectral density. All peaks have the same shape, the number of peaks scales with the number of elements, and their height scales inversely with the number of elements. Some exemplary spectra are shown in Figs. 1(b), 1(d), 1(f), and 1(h).

\section{RINGS OF DETERMINISTIC CHAOTIC SYSTEMS}

To test the generality of these results we compare the correlation properties of a unidirectional ring of linear maps to those of different deterministic chaotic systems coupled with sufficiently long interaction delays. We first evaluate delay-coupled Stuart-Landau oscillators. Since it is the normal form of a limit-cycle oscillator close to threshold, the behavior of Stuart-Landau oscillators is generic for coupled oscillator systems. In particular, Stuart-Landau oscillators show some similarities to semiconductor lasers subject to interaction delays [22]. Moreover, in-phase and out-of-phase states can be easily mapped onto each other by adjusting the coupling phase since the system is rotationally symmetric. A unidirectional ring of $N$ Stuart-Landau oscillators is modeled by

$$
\dot{z}_{n}=z_{i}\left(1-z_{n}\right)^{2}+i \beta z_{n}\left|z_{n}\right|^{2}+\kappa z_{n-1}(t-\tau),
$$

with $1 \leqslant n \leqslant N$ and $0 \equiv N$. We choose the complex coupling strength $\kappa$, the coupling delay $\tau$, and the amplitudephase coupling $\beta$ such that the nonlinear oscillators behave chaotically.

In Fig. 2 the real and imaginary parts of the autocorrelation functions of a single oscillator with positive [Fig. 2(a)] and negative [Fig. 2(b)] feedback are shown. It is clear that

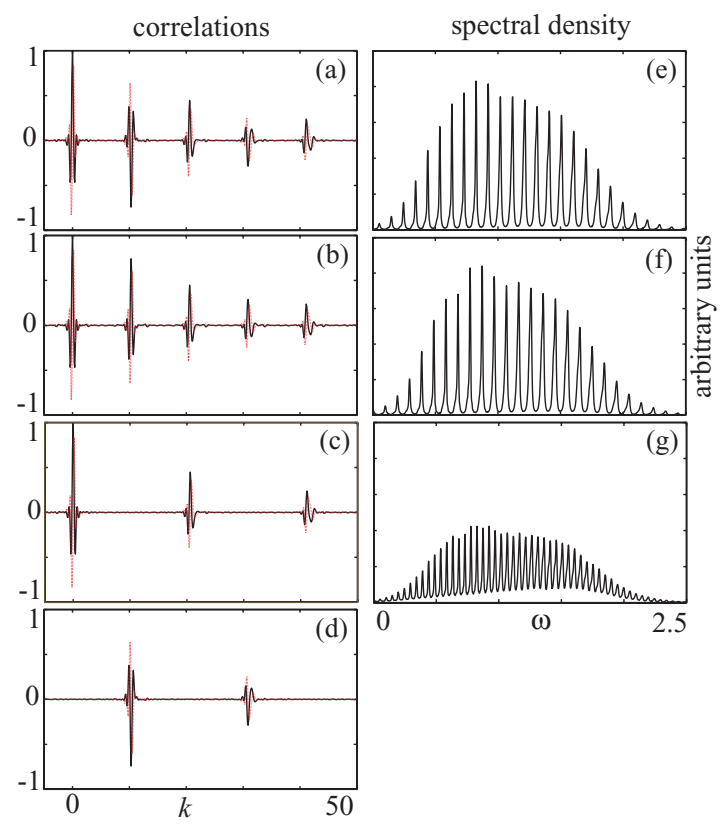

FIG. 2. (Color online) Panels (a) and (e) and panels (b) and (f) show, respectively, the autocorrelation function and spectral densities of a single Stuart-Landau oscillator with positive and negative delayed feedback (black solid and thin red (gray) dashed lines for real and imaginary parts, respectively). Panels (c) and (d) contain the complex autocorrelation and cross correlation functions of two coupled StuartLandau oscillators, respectively. Panel (g) shows the density of two coupled oscillators. Note that the spectra are plotted on a linear scale. The parameters are $\beta=5, \kappa= \pm 1.5$, and $\tau=10$.

the odd-numbered peaks have opposite phases, while the even-numbered peaks have the same phases. Comparing the autocorrelation [Fig. 2(c)] of a mutually coupled system with those of the single oscillators, we find that it is almost an exact average of the system with a positive feedback phase and the system with a negative feedback phase. The cross correlation [Fig. 2(d)] of two mutually coupled Stuart-Landau oscillators shows excellent agreement with the difference of the autocorrelations of the positive and negative feedback systems, as predicted by Eqs. (5).

Figures 2(e) and 2(f) show the spectral densities of these systems with opposite feedback phase. We find that they show a broadband spectrum centered around the frequency of the uncoupled oscillator. As for linear maps, the discrete delay peaks are shifted in both spectra. The spectral density of two coupled oscillators [Fig. 2(g)] shows twice as many discrete peaks, which are half as high as in the single system; the quantitative agreement with Eq. (9) for stochastic maps is excellent.

Also in unidirectional rings of Stuart-Landau oscillators with more elements we find that the covariance functions exhibit the same feature: The network covariance functions are an average of the in-phase and out-of-phase covariance functions, just like the spectral densities. Replacing the Stuart-Landau oscillators by Lang-Kobayashi lasers, we find that Eqs. (4) and (9) still hold.

In most chaotic systems it is not possible to calculate the out-of-phase dynamics by means of a single system by adjusting the coupling parameters. Nevertheless, we find the 
same pattern for the covariance functions and spectra in unidirectional rings of coupled tent maps as in stochastic maps: The variance and the shape of the maxima do not change with the number of elements; in the covariance functions the number of peaks decreases with the number of elements and in the spectral density the number of discrete peaks scales linearly, but their height scales inversely with the number of elements.

\section{DISCUSSION}

In conclusion, we have found a scaling relation for linear stochastic maps Eq. (4) relating the dynamics of a ring to different single units with delayed feedback. These different units correspond to in-phase and out-of-phase oscillations in the ring; the dynamics of the ring elements can be seen as a sum of in-phase and out-of-phase contributions. In particular for a unidirectional ring, this results in a characteristic pattern for the covariance functions and spectra: As elements are added in the ring, some extrema cancel in the covariance functions and extra maxima appear in the spectral density. However, the variance of the units and the shape of the maxima do not change with the size of the ring. The scaling law also allows us to infer from a local measure, such as the autocorrelation or power spectrum of a single unit in the network, global properties such as the total round-trip delay and the number of elements in the network.

Surprisingly, we find that unidirectional rings of chaotic deterministic elements behave in the same way as linear stochastic systems. We find that the linear relations for linear maps Eqs. (6) and (9) apply for nonlinear systems as well: When coupling chaotic elements in a unidirectional ring, some statistical properties, such as the autocorrelation function and spectral density, can be seen as the sum of an in-phase and multiple out-of-phase synchronized states. In this sense, nonlinear elements coupled in a unidirectional ring behave like coupled linear systems when the delay is sufficiently long. We found such a pattern for systems in which the chaotic behavior is induced by the delayed coupling, such as Stuart-Landau oscillators, as well as for systems that autonomously exhibit chaotic behavior, such as tent maps.

However, deterministic chaotic systems coupled in a unidirectional ring can show so-called generalized synchronization of leader-laggard type, while coupled linear systems cannot show any form of synchrony and only reflect the coupling topology. Still, linear maps and chaotic systems show the same correlation patterns irrespective of whether this chaotic behavior is already present in the individual elements or induced by the delayed coupling. This suggests that in a delay-coupled unidirectional ring the correlation properties are dominated by the topology.

An underlying reason might be the symmetry between in-phase and out-of-phase rotations in a fully symmetric unidirectional ring, which is induced by the coupling delay.
For rotationally symmetric systems such as Stuart-Landau oscillators, the manifolds associated with these different oscillation patterns can be calculated explicitly; they differ only by a coupling phase factor. If the delay is sufficiently long such that the delayed chaotic signal can be seen as an independent drive, one can argue that the coupling phase no longer plays a qualitative role in the dynamics. Therefore, in-phase and out-of-phase manifolds share similar dynamical properties and similar bifurcation patterns [16]. This could explain why in-phase and out-of-phase rotations are equally represented in the spectral density of a unidirectional ring. The condition of long coupling delays, which arises from theoretical results, is not so strict; in our numerical simulations we retrieve the linear pattern for coupling delays, which are only a few times the internal time scale of our system. We note properties similar to the periodical regime in rings of delay-coupled oscillators, in which several in-phase and out-of-phase orbits coexist, exhibiting similar characteristics and stability properties [23-25].

In this sense, the correlation pattern and spectral density show the identifying characteristic of the coupling topology, but due to identical synchronization effects it can be altered (i.e., out-of-phase oscillations can be suppressed, as the synchronization manifold becomes transversely stable). In particular, the correlation pattern associated with leader-laggard behavior, as it is observed for two mutually delay-coupled elements (for example, lasers [3]), results from the coupling topology and does not necessarily imply a synchronization effect.

We expect such a linear correlation pattern in networks with long coupling delays, where all directions have similar stability properties, and identical synchronization cannot occur. This is not only the case in unidirectional rings, but it can also occur in networks of strongly chaotic elements [26]. We found indeed that the correlation patterns of three mutually linearly coupled tent maps show excellent agreement with those of coupled stochastic maps. We believe that our findings open perspectives for the understanding of the dynamics of delaycoupled networks that do not show identical synchronization.

\section{ACKNOWLEDGMENTS}

O.D. acknowledges the Research Foundation Flanders (Fonds Wetenschappelijk Onderzoek Vlaanderen) for a fellowship and for project support. R.V. acknowledges the support from the Hertie Foundation. This work was partially supported by the Interuniversity Attraction Poles program of the Belgian Science Policy Office, under Grant No. IAP VI-10 “photonics@be"; by Ministerio de Ciencia e Innovacion (Spain), under Project DeCoDicA (No. TEC2009-14101); and by Project PHOCUS (EU Future and Emerging Technologies Open Grant No. 240763). L. Keuninckx, L. Lean, and G. Van der Sande are acknowledged for helpful discussions.
[1] T. Erneux, Applied Delay Differential Equations (Springer, New York, 2009).

[2] Philos. Trans. R. Soc. London Ser. A 368, 303 (2010), Special issue on Delayed Complex Systems, edited by W. Just, A. Pelster, M. Schanz, and E. Schöll.
[3] T. Heil, I. Fischer, W. Elsäßer, J. Mulet, and C. R. Mirasso, Phys. Rev. Lett. 86, 795 (2001).

[4] M. Y. Kim, R. Roy, J. L. Aron, T. W. Carr, and I. B. Schwartz, Phys. Rev. Lett. 94, 088101 (2005).

[5] P. Nelson and A. Perelson, Math. Biosci. 179, 73 (2002). 
[6] G. Orosz, R. E. Wilson, R. Szalai, and G. Stépan, Phys. Rev. E 80, 046205 (2009).

[7] W. Wang and J. Slotine, IEEE Trans. Automat. Contr. 51, 712717 (2006).

[8] W. Mather, M. R. Bennett, J. Hasty, and L. S. Tsimring, Phys. Rev. Lett. 102, 068105 (2009).

[9] L. Chen and K. Aihara, IEEE Trans. Circuits Syst. I 49, 602 (2002).

[10] Philos. Trans. R. Soc. London Ser. A 367, 1059 (2009), Special issue on Delay Effects in Brain Dynamics, edited by G. Stepan.

[11] A. Argyris, D. Syvridis, L. Larger, V. Annovazzi-Lodi, P. Colet, I. Fischer, J. Garcia-Ojalvo, C. Mirasso, L. Pesquera, and K. A. Shore, Nature 438, 343 (2005).

[12] L. Appeltant, M. Soriano, G. V. der Sande, J. Danckaert, S. Massar, J. Dambre, B. Schrauwen, C. Mirasso, and I. Fischer, Nature Commun. 2, 468 (2011).

[13] F. M. Atay, J. Jost, and A. Wende, Phys. Rev. Lett. 92, 144101 (2004).

[14] I. Fischer, R. Vicente, J. M. Buldu, M. Peil, C. R. Mirasso, M. C. Torrent, and J. Garcia-Ojalvo, Phys. Rev. Lett. 97, 123902 (2006).

[15] D. V. Senthilkumar, J. Kurths, and M. Lakshmanan, Phys. Rev. E 79, 066208 (2009).
[16] V. Flunkert, S. Yanchuk, T. Dahms, and E. Schöll, Phys. Rev. Lett. 105, 254101 (2010).

[17] A. Englert, W. Kinzel, Y. Aviad, M. Butkovski, I. Reidler, M. Zigzag, I. Kanter, and M. Rosenbluh, Phys. Rev. Lett. 104, 114102 (2010).

[18] B. Ravoori, A. B. Cohen, J. Sun, A. E. Motter, T. E. Murphy, and R. Roy, Phys. Rev. Lett. 107, 034102 (2011).

[19] L. Illing, C. D. Panda, and L. Shareshian, Phys. Rev. E 84, 016213 (2011).

[20] G. Van der Sande, M. C. Soriano, I. Fischer, and C. R. Mirasso, Phys. Rev. E 77, 055202 (2008).

[21] W.-X. Wang, Q. Chen, L. Huang, Y.-C. Lai, and M. A. F. Harrison, Phys. Rev. E 80, 016116 (2009).

[22] D. Pieroux and P. Mandel, Phys. Rev. E 68, 036204 (2003).

[23] P. Perlikowski, S. Yanchuk, O. V. Popovych, and P. A. Tass, Phys. Rev. E 82, 036208 (2010).

[24] C. U. Choe, T. Dahms, P. Hövel, and E. Schöll, Phys. Rev. E 81, 025205(R) (2010).

[25] O. D'Huys, R. Vicente, T. Erneux, J. Danckaert, and I. Fischer, Chaos 18, 037116 (2008).

[26] S. Heiligenthal, T. Dahms, S. Yanchuk, T. Jüngling, V. Flunkert, I. Kanter, E. Schöll, and W. Kinzel, Phys. Rev. Lett. 107, 234102 (2011). 